\title{
In-Situ TEM Investigation of Controlled VLS Silicon Nanowire Device Formation and Characterization
}

\author{
S.B. Alam ${ }^{1}$, F. Panciera ${ }^{2,3}$, M.M Norton ${ }^{4}$, O. Hansen ${ }^{1,5}$, F.M. Ross ${ }^{2}$ and K. Mølhave ${ }^{1 *}$ \\ 1. Dept. of Micro and Nanotechnology, Technical University of Denmark (DTU), Denmark. \\ 2. IBM Research Division, T. J. Watson Research Center, Yorktown Heights, NY, USA. \\ 3. Dept. of Engineering, University of Cambridge, Cambridge, United Kingdom. \\ 4. Dept. of Mechanical Engineering and Applied Mechanics, University of Pennsylvania, PA, USA. \\ 5. CINF-Center for Individual Nanoparticle Functionality, DTU.*krmo@nanotech.dtu.dk.
}

Detailed understanding of complex nanoscale processes can be achieved by in-situ TEM by using a microchip system that can provide full control of process parameters such as temperature, applied bias potentials and currents [1]. We here review recent results on joule heated microcantilevers used for gas phase in-situ TEM studies of silicon nanowire (SiNW) devices.

Loop shaped microcantilevers made from doped monocrystalline silicon and extending over the edge of a microchip can be used as resistive heating platforms for VLS nanowire growth under TEM or optical imaging [2] as illustrated in Fig 1. The cantilevers allow direct imaging of coarsening processes involving the AuSi eutectic liquid, as well as nanowire nucleation and growth. The growth rate can be used as a local temperature measurement at known disilane gas pressure in the TEM [3]. By growing SiNWs to form a bridging connection between two adjacent cantilevers, electrically connected SiNW devices can be formed and their electrical properties characterized in-situ [4].

As the growing SiNW approaches to within $\sim 100 \mathrm{~nm}$ proximity to the adjacent cantilever, applying a potential difference of 10-100 V will deform the eutectic droplet into an ellipsoidal shape due to the electrical field [6]. The deformed droplet alters the SiNW growth, changing its diameter as the droplet changes contact angle to the SiNW, or modifying the growth direction towards the adjacent cantilever. By analysing the aspect ratio of the deforming droplet as the field strength is varied, and fitting to numerical predictions of the droplet deformation, we can measure the surface tension under growth conditions. The result, $0.55 \mathrm{~J} / \mathrm{m}^{2}$, is only about half of the earlier literature values [6].

Upon growing into contact with the adjacent cantilever, the AuSi eutectic is displaced and crystalline silicon is deposited. Various contact geometries can be created, depending on the conditions that determine the $\mathrm{Au}$ migration rate away from the contact site and the silicon deposition rate [5]. Electromigration, thermomigration, as well as Ostwald ripening effects can influence the final contact shape. A simple model for the deposition rate of silicon includes both the disilane gas phase supply and the removal of $\mathrm{Au}$ (by electromigration or Ostwald ripening) from the AuSi, which causes $\mathrm{Si}$ to segregate and deposit. The temperature is the most important variable [5], but if a bias voltage is applied across the SiNW during contact formation, electromigration may become dominant and can be used to controllably create gaps or necks with reduced diameter at the contact site [5].

A detailed analysis of the contact formation dynamics allows correlation of the migration of gold from the AuSi droplet with the current passing through the nanowire. Figures 2 and 3 show such a study where we have placed an eutectic between two nanowires by electro/thermomigration.In this case we turn off the disilane supply to halt the contribution to the growth of Si from the gas phase supply. The observed deposition of $\mathrm{Si}$ in Figures 2 and 3 is then due to the removal of $\mathrm{Au}$ from the AuSi by electromigration. From the eutectic volume we can measure the gold electromigration rate in terms of $\mathrm{Au}$ volume per coulomb. In this case the result is $\sim 10^{8} \mathrm{~nm}^{3} / \mathrm{C}$. This type of measurement allows the 
nanowire/cantilever junction to be designed by appropriate choice of growth conditions and electromigration, in principle enabling controlled electrical properties of the final nanowire device [7]. References:

[1] F. (Feng) Tao et al., Science, vol. 331, Jan. 2011, pp. 171-174.

[2] K. Molhave et al., Small, vol. 4, Oct. 2008, pp. 1741-1746.

[3] C. Kallesøe et al., Small, vol. 6, 2010, pp. 2058-2064.

[4] C. Kallesøe et al., Nano Letters, vol. 12, Jun. 2012, pp. 2965-2970.

[5] S.B. Alam et al., Nano Letters, vol. 15, Oct. 2015, pp. 6535-6541.

[6] F. Panciera et al., Nature Communications, 7:12271 doi: 10.1038/ncomms12271 (2016).

[7] Supported by ERC Grant 279342: InSituNANO and EPSRC Grant EP/P005152/1 (F.P., S.H.), by the National Science Foundation CBET 1066573 and the Nano/Bio Interface Center through the National Science Foundation NSEC DMR08-32802 (M.M.N.), and The Danish Council for Independent Research, Case No.10-083797 (S.B.A., K.M.). The authors acknowledge Jerry D. Tersoff and Haim H. Bau for fruitful discussions and Mark C. Reuter, Arthur W. Ellis and Eric Jensen for technical support.
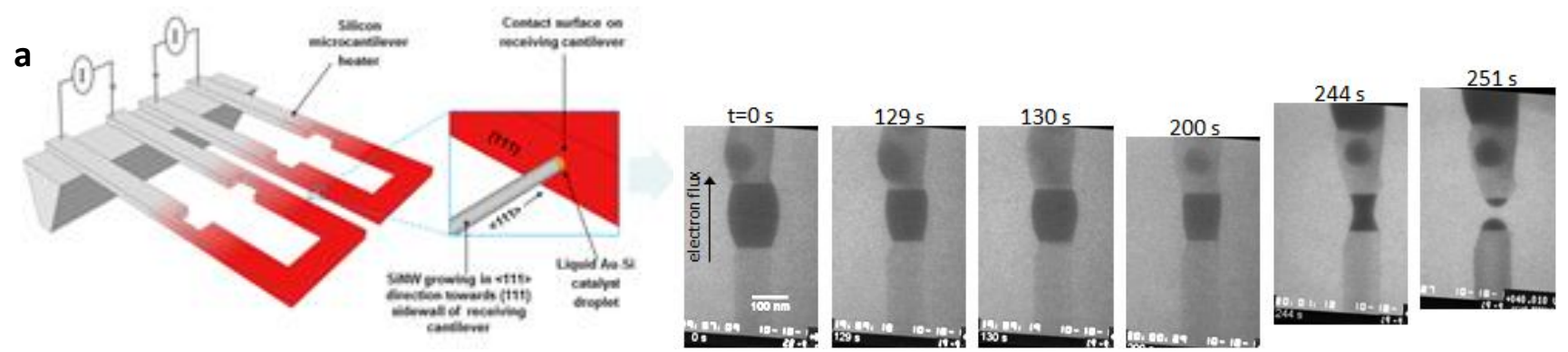

Figure 1: (a) Schematic illustration of the cantilever setup. (b) TEM image series of a silicon nanowire suspended between cantilevers, where the AuSi droplet has been moved to the middle by electro/thermomigration and the disilane gas supply turned off to halt growth for the study in Fig 2.
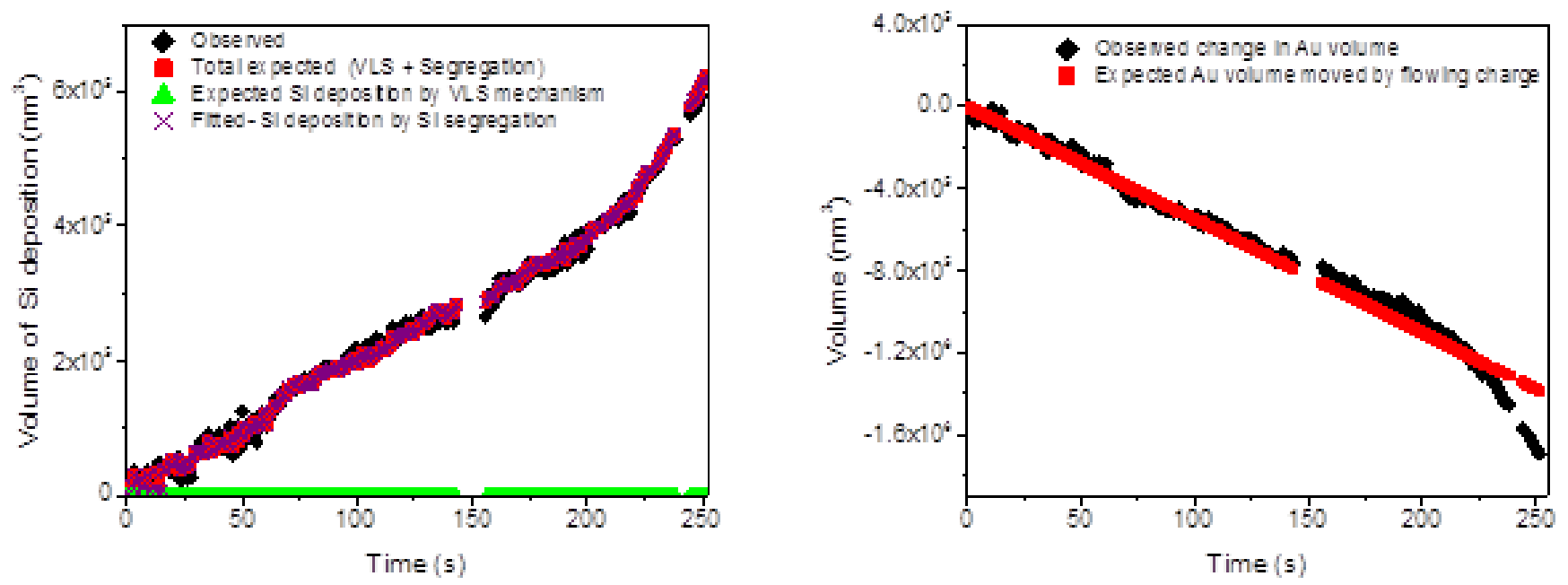

Figure 2: (a) Modelling the silicon deposition as a sum of gas phase supply through the observed eutectic surface area plus the silicon segregated from the AuSi closely fits the observed volumes giving as fitting parameter the $\mathrm{Si}$ composition in the AuSi hyper-eutectic to be $28 \%$. This is close to the expected $23 \%$ from equilibrium conditions at $475^{\circ} \mathrm{C}$. (b) $\mathrm{Au}$ removed by electromigration, with a straight line fit of the observed volume loss vs. charge passed that yields a fitting constant of $-1.67 \times 10^{8}$ $\mathrm{nm}^{3} / \mathrm{C}$ obtained by minimizing the sum of squared error. 